\title{
Early Enteral Nutrition Is Associated With Lower Mortality in Critically III Children
}

早期肠内营养与危重患儿的低病死率相关

Theresa A. Mikhailov, MD, PhD $1, \underline{2}$

Evelyn M. Kuhn, PhD2

Jennifer Manzi, CPNP-AC/PC르는

Melissa Christensen, BS 4

Maureen Collins, MS, RD?

Ann-Marie Brown, MSN, CPNP-AC/PC, CCRN, FCCM $\underline{5}$

Ronald Dechert, RRT, DPH $\underline{6}$

Matthew C. Scanlon, MD1, 2

Martin K. Wakeham, MD $\underline{1}, \underline{2}$

Praveen S. Goday, MBBS, CNSC 7

${ }^{1}$ Pediatric Critical Care, Medical College of Wisconsin, Milwaukee, Wisconsin

${ }^{2}$ Children's Hospital of Wisconsin, Milwaukee, Wisconsin

${ }^{3}$ Department of Pediatrics, Medical College of Wisconsin, Milwaukee, Wisconsin

${ }^{4}$ Department of Surgery, Medical College of Wisconsin, Milwaukee, Wisconsin

${ }^{5}$ Division of Critical Care, Akron Children's Hospital, Akron, Ohio

${ }^{6}$ University of Michigan Hospital and Health Center, Ann Arbor, Michigan

${ }^{7}$ Pediatric Gastroenterology and Nutrition, Medical College of Wisconsin, Milwaukee, Wisconsin

Theresa A. Mikhailov, MD, PhD, Pediatric Critical Care, Medical College of Wisconsin, 9000

West Wisconsin Avenue, Milwaukee, WI 53226, USA. Email:tmikhail@mcw.edu

\section{摘要}

背景: 本研究的目的是研究早期肠内营养 (early enteral nutrition, EEN) 与危重病患儿的发病率和 死亡率之间的关系。早期肠内营养定义为入院 48 小时内提供目标热卡的 $25 \%$ 。方法: 在 12 个儿科 重症监护病房（PICU）进行多中心的回顾性研究。研究对象为2007-2008年间，在PICU住院时间 (length of stay, LOS) $\geq 96$ 小时, 年龄 1 个月至18岁患儿。我们获得了患儿的人口统计学资料、体 重、儿科死亡率指数2（PIM2）评分、住院时间（LOS）、机械通气时间（MV）、死亡率和入院 后的前 4 天营养摄入量数据。结果： 5105 例患儿（男性 $53.8 \%$; 平均年龄 2.4 岁），死亡率为 $5.3 \%$ 。 接受早期肠内营养治疗（EEN）的患儿为 $27.1 \%$ 。接受EEN治疗的患儿比未接受治疗的患儿的死亡 率低（优势比, $0.51 ; 95 \%$ 置信区间, 0.34-0.76; P =.001 [调整倾向得分, PIM2得分、年龄、中 心因素后]）。接受与没有接受EEN治疗的患儿比较, 调整PIM2得分、年龄和中心因素后, 发现 PICU住院时间没有差异 $(\mathrm{P}=0.59)$; 而在机械通气时间（MV）上, 接受EEN 治疗的患儿比未接 受治疗的患儿MV时间长, 但差异无显著性 $(\mathrm{P}=0.058)$ 。结论: 在 PICU住院时间 $\geq 96$ 小时的患儿 中, EEN与患儿死亡率降低显著相关。接受EEN的患儿, LOS和MV时间稍长, 但差异无统计学意 义。 